ARTICLE

Received 1 Dec 2015 | Accepted 2 Aug 2016 | Published 29 Sep $2016 \quad$ DOI: 10.1038/ncomms12811

\title{
Topographic generation of submesoscale centrifugal instability and energy dissipation
}

Jonathan Gula1, M. Jeroen Molemaker ${ }^{1,2} \&$ James C. McWilliams ${ }^{2}$

Most of the ocean kinetic energy is contained in the large scale currents and the vigorous geostrophic eddy field, at horizontal scales of order $100 \mathrm{~km}$. To achieve equilibrium the geostrophic currents must viscously dissipate their kinetic energy at much smaller scale. However, geostrophic turbulence is characterized by an inverse cascade of energy towards larger scale, and the pathways of energy toward dissipation are still in question. Here, we present a mechanism, in the context of the Gulf Stream, where energy is transferred from the geostrophic flow to submesoscale wakes through anticyclonic vertical vorticity generation in the bottom boundary layer. The submesoscale turbulence leads to elevated local dissipation and mixing outside the oceanic boundary layers. This process is generic for boundary slope currents that flow in the direction of Kelvin wave propagation. Topographic generation of submesoscale flows potentially provides a new and significant route to energy dissipation for geostrophic flows.

\footnotetext{
${ }^{1}$ Univ. Brest, CNRS, IRD, Ifremer, Laboratoire d'Océanographie Physique et Spatiale (LOPS), IUEM, Brest 29280, France. ${ }^{2}$ Department of Atmospheric and Oceanic Science, University of California, Los Angeles, California 90095-1565, USA. Correspondence and requests for materials should be addressed to J.G. (email: jonathan.gula@univ-brest.fr).
} 
$\mathrm{D}$ ifferent mechanisms can extract energy from geostrophic flows and transfer it to unbalanced motions, from where it may be cascaded to smallest scale where irreversible molecular mixing takes place ${ }^{1,2}$. One pathway has been identified in the oceanic surface layer where frontogenesis in the intense fronts $^{3}$ and filaments ${ }^{4}$ is an efficient way to transfer energy from the mesoscale to unbalanced motions. Different type of ageostrophic instabilities can provide a direct route from balanced mesoscale dynamics to unbalanced submesoscale behaviours ${ }^{5}$. In particular, atmospheric forcing at fronts can make the flow unstable to symmetric instability and initiate a forward cascade of energy down to dissipation ${ }^{6-8}$. The nearinertial wave field excited by the wind may also extract significant energy from the geostrophic flows ${ }^{9,10}$. Another pathway lies at the bottom of the ocean where interactions of geostrophic flows with small-scale bottom topography generate internal gravity waves that can break and dissipate energy at small scales ${ }^{11}$. There is a more recent and much less studied mechanism associated with the generation of submesoscale flows by interaction of geostrophic flows with steep topographic slopes ${ }^{2-14}$.

Most of the mean kinetic energy (MKE) is concentrated in the intense western boundary currents, such as the Gulf Stream, and they are known to be sites of elevated eddy energy dissipation ${ }^{15}$. It is, thus, an ideal place to study the interaction of geostrophic flows with topography and investigate the possible impact of topography on energy dissipation and mixing.

To this end, we perform realistic simulations of the Gulf Stream using very high horizontal and vertical resolutions ( $\Delta x=200 \mathrm{~m}$, see 'Methods' section) that allow us to adequately resolve submesoscale motions in the range $1-30 \mathrm{~km}$. The results show that the interaction of the incident geostrophic flow with topography can generate unbalanced submesoscale turbulence that leads to elevated local dissipation and mixing outside the oceanic boundary layers. Energy dissipated locally through the process is comparable to the energy dissipated in intense surface frontogenetic regions. Interior regions of high energy dissipation, with the implication of strong vertical mixing of material properties across stably stratified isopycnal surfaces, is of great interest for its potential significance for the energy budget of the general circulation and the maintenance of the density stratification.

\section{Results}

Topographic vorticity generation. The Gulf Stream strongly interacts with topography as it flows through the Florida Straits. The Stream is constrained by the Florida Shelf on its cyclonic side and the Great Bahama Bank and the Little Bahama Bank on its anticyclonic side (Fig. 1a). On the cyclonic side, flow-topography interactions lead to barotropic shear instability and formation of streets of submesoscale vortices ${ }^{14}$. The topographic drag against the slope amplifies the cyclonic shear by generating large positive vertical vorticity values within the sloped turbulent bottom boundary layer. The flow partially separates from the topography downstream from the Straits, and due to the large horizontal velocity shear, often becomes unstable to submesoscale barotropic instability, rolls up and forms streets of submesoscale vortices. Submesoscale cyclones will also similarly be created at any place where a current interacts with the topography on its left (in the northern hemisphere), which corresponds to a current that flows in the direction opposite to the Kelvin wave propagation direction.

On the anticyclonic side of the Stream there is a similar topographic vorticity generation, but with the opposite sign (Fig. 1a,b). The topographic drag against the slope amplifies the anticyclonic shear and generates large negative vertical vorticity values. Following the sequence of processes described in the context of the California Undercurrent ${ }^{12}$, relative vorticity can locally become much smaller than $-f$, where $f$ is the Coriolis frequency, and Ertel's potential vorticity (PV, see 'Methods' section) can become negative, which is a criterion for ageostrophic centrifugal instability ${ }^{16}$.

Negative values of PV can be spotted in different regions (Fig. 2) where the flow strongly interacts with the topography: (i) along the slope of the Great Bahama Bank, downstream of the Bimini Islands $\left(26^{\circ} \mathrm{N}, 79.3^{\circ} \mathrm{W}\right)$; (ii) along the western part of the Little Bahama Bank; (iii) at the southern tip of the Grand Bahama

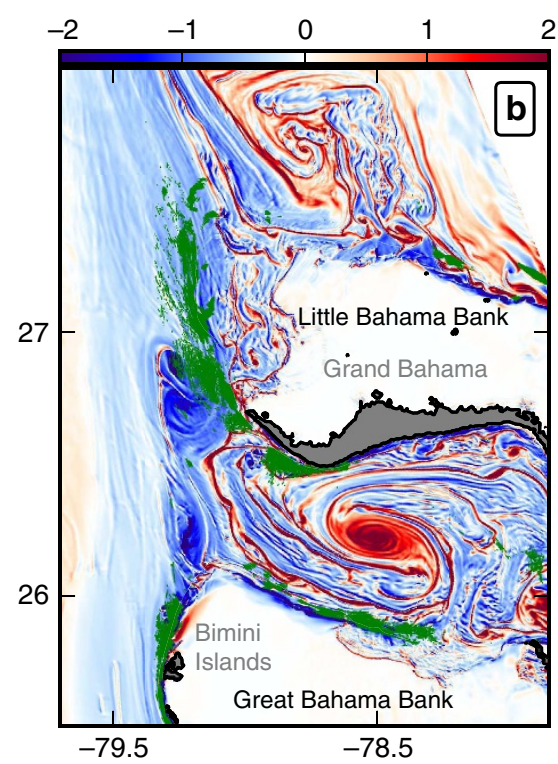

Figure 1 | Topographic generation of negative vorticity along the Bahamas Banks. (a) Instantaneous surface relative vorticity $\zeta=v_{x}-u_{y}$, normalized by $f_{\text {, }}$ for the Gulf Stream along the southeast US seaboard as simulated by ROMS. The boundaries of the successive nested domains ( $\Delta x=2.5,0.75$ and $0.20 \mathrm{~km}$ ) are delineated by thick grey lines. (b) Zoomed-in view of the Gulf Stream along the Bahamas Banks. Green contours show region of high energy dissipation (depth-integrated energy dissipation $\langle\epsilon\rangle>2 \times 10^{-4} \mathrm{~W} \mathrm{~m} \mathrm{~kg}^{-1}$ ). Localized regions with negative vorticity and high energy dissipation rates are created by the currents flowing along the topographic slopes of the Bahamas Banks. 


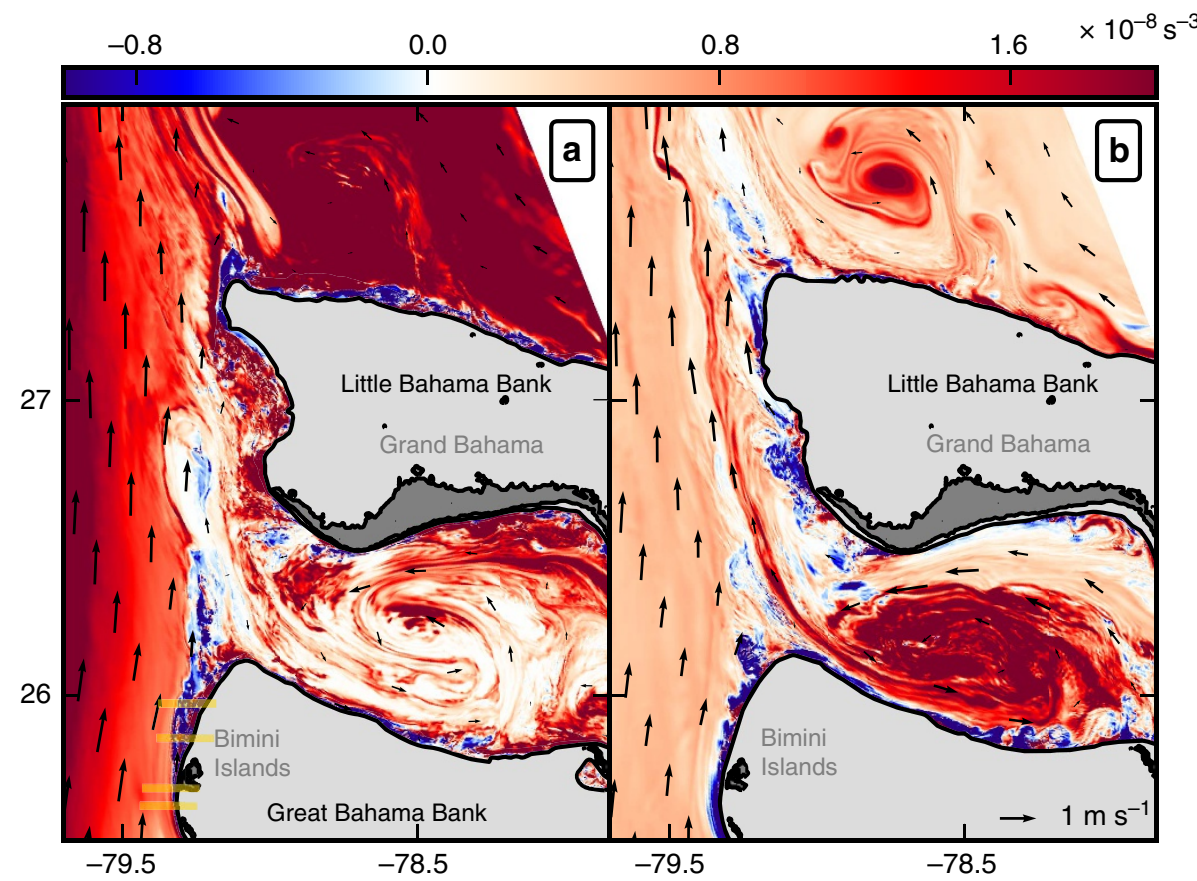

Figure 2 | Generation of negative potential vorticity and centrifugal instability. Snapshots of PV (in $10^{-8} \mathrm{~s}^{-3}$ ) and velocity (vectors) at (a) $z=-50 \mathrm{~m}$ and (b) $z=-100 \mathrm{~m}$. Localized regions with highly negative PV are created by the currents flowing along the topographic slopes of the Bahamas Banks.

Island $\left(26.5^{\circ} \mathrm{N}, 78.8^{\circ} \mathrm{W}\right)$; and (iv) on the northern side of the Great Bahama Bank $\left(26^{\circ} \mathrm{N}, 78.5^{\circ} \mathrm{W}\right)$. The last two locations correspond to the interaction of a cyclonic eddy trapped in the Northwest Providence Channel, which separates the Little Bahama Bank from the Great Bahama Bank (Fig. 1b). It is a recurrent and observed feature of the local circulation ${ }^{17}$. PV at all the aforementioned locations is dominated by its vertical component. The negative values are obtained through a combination of stable stratification and strong negative values of vertical vorticity. Negative values of PV also coincide with high energy dissipation (Fig. 1b).

The sequence of processes consists of three different steps: negative PV generation within the bottom boundary layer; separation of the negative PV strip from the slope; and intense small-scale instabilities and energy dissipation in the separated wake (Fig. 3). The negative PV sheet forms along the bottom slope where vertical and horizontal scales of the boundary sheared layer are locally about 50 and $500 \mathrm{~m}$ with a cross-shelf bottom slope as high as 0.1 along the Grand Bahamas Bank (Fig. 3a). Given a current of $0.5-1 \mathrm{~m} \mathrm{~s}^{-1}$, we can evaluate the scale of the vertical vorticity in the boundary layer as $\mathcal{O}(10 f-20 f)$, which is consistent with the instantaneous maximum relative vorticity values in the simulation. The model has 100 levels in the vertical with grid stretching near the bottom such that the vertical resolution does not exceed $2 \mathrm{~m}$ in the boundary layer in the region of strong topographic vorticity generation.

Centrifugal instability. A change in the sign of PV is a sufficient condition for instability in an unbounded fluid ${ }^{16}$. The instabilities that arise can extract their kinetic energy from the eddy potential energy (EPE) through the vertical buoyancy flux (VBF, see 'Methods' section) or from the MKE through a combination of the vertical shear production term (VRS) and the horizontal shear production term (HRS). Centrifugal (or inertial) instability is triggered when the relative vorticity is smaller than $-f$ and extracts its energy mostly from the lateral shear $(\text { HRS }>0)^{18}$. The positive conversions from mean to eddy kinetic energy (EKE) seen in the regions of sustained negative potential vorticity generation (Fig. $4 \mathrm{~b}, \mathrm{~d}$ ) are due to the lateral shear. The vertical shear production term is an order of magnitude smaller (Supplementary Fig. 1). The vertical buoyancy flux term (Fig. 4e) is negative in the region of instability. This is indicative of centrifugal instability, and it is consistent with the results obtained from comparisons of model and linear instability analysis for the California Undercurrent ${ }^{13}$.

The horizontal shear production term has a two-signed pattern in regions of topographic interactions. The energy conversion is from eddy to mean kinetic energy on the upstream sides where the bottom drag over the slope intensifies the mean horizontal velocity shear and suppresses cross-stream velocity perturbations growth. The energy conversion is from mean to eddy kinetic energy downstream from the topographic features, indicative of instability and eddy generation.

Energy dissipation. Locations of sustained generation of centrifugal instability correspond to EKE dissipation maxima (Fig. 4a,c). Where the current directly interacts with the topography (that is, upstream from the Bimini Islands), there is dissipation of MKE due to the vertical mixing occurring in the bottom boundary layer that is directly triggered by the bottom drag (Supplementary Fig. 2). Downstream from the generation sites the dissipation is also dominated by the vertical mixing term, but in the ocean interior, outside of the surface and bottom boundary layers, where it represents the parameterization of small Richardson number processes and static instabilities ${ }^{19}$. The vertically integrated dissipation rates of EKE reach values up to $8 \times 10^{-4} \mathrm{~W} \mathrm{~m} \mathrm{~kg}^{-1}$ instantaneously at $26^{\circ} \mathrm{N}$ following separation of the negative PV strip from the slope (Fig. 3b). It is of the same order as the dissipation rates observed in an intense surface front within the Kuroshio Current ${ }^{8}$ integrated over the mixed-layer. 


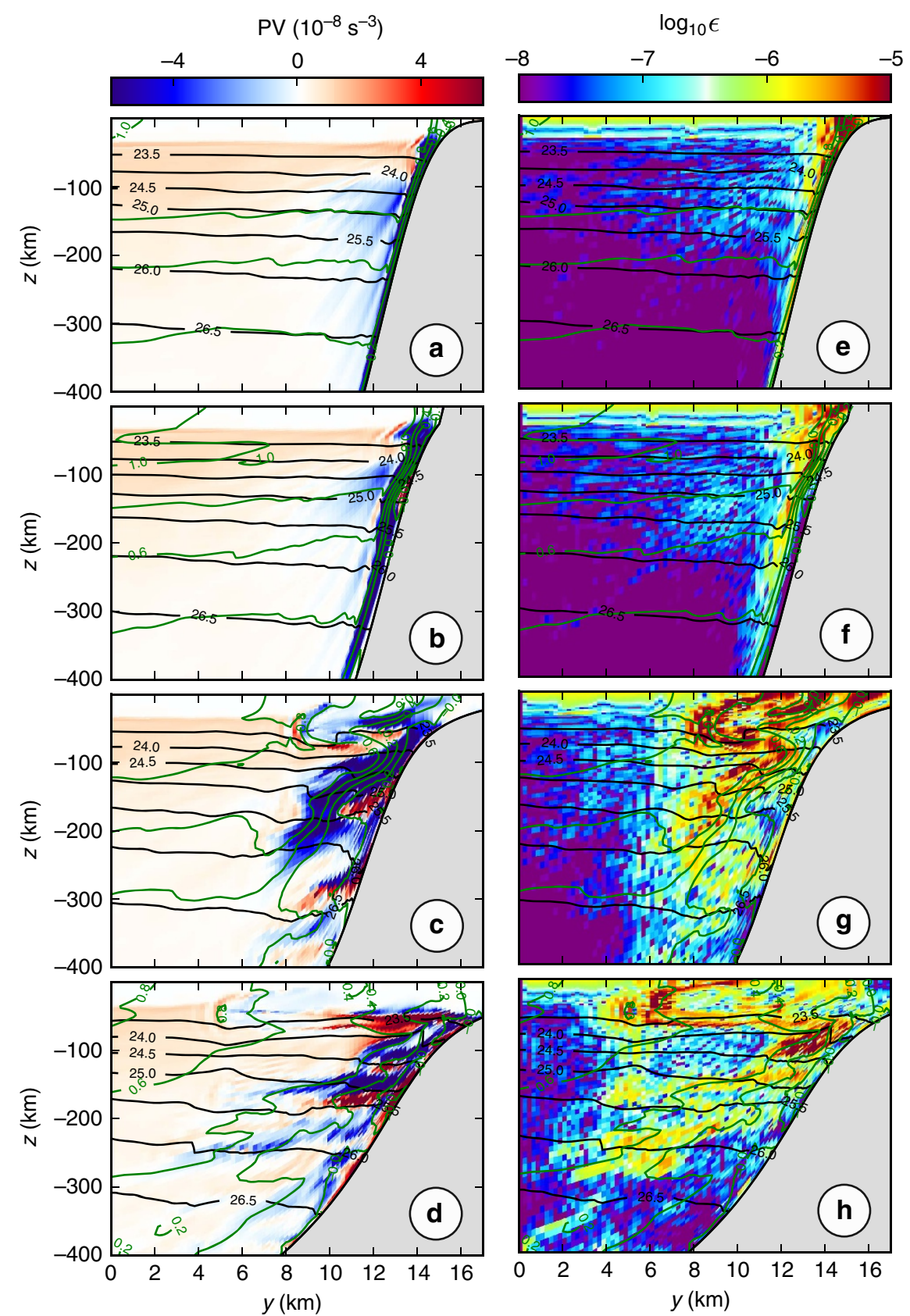

Figure 3 | Submesoscale centrifugal instability of the boundary layer. (a-d) Vertical sections of potential vorticity $\left(P V\right.$, in $\left.10^{-8} S^{-3}\right)$ and $\left(\mathbf{e}^{-h}\right)$ energy dissipation $\epsilon\left(\mathrm{m}^{2} \mathrm{~s}^{-3}\right)$ from upstream to downstream along the Great Bahamas Banks (see yellow lines in Fig. 2) showing (a,e) PV generation, (b,f) separation from the slope, and $(\mathbf{c}, \mathbf{d}, \mathbf{g}, \mathbf{h})$ centrifugal instability in the wake. Density is shown in black contours with an interval of $0.5 \mathrm{~kg} \mathrm{~m}^{-3}$ and alongslope velocity is shown in green contours with an interval of $0.2 \mathrm{~m} \mathrm{~s}^{-1}$.

The EKE dissipation rates averaged over a 3 months period (Fig. 4c) have values up to $2 \times 10^{-4} \mathrm{~W} \mathrm{~m} \mathrm{~kg}^{-1}$, which are only slightly smaller than the instantaneous values. This shows how remarkably sustained are the topographic generation of vorticity and the subsequent centrifugal instability. The kinetic energy dissipation rates are roughly an order of magnitude larger than the dissipation rates due to the same mechanisms in the California Undercurrent ${ }^{12}$, where the velocity shears are weaker. The rate of conversion from mean to eddy kinetic energy downstream from the Bimini Islands reaches values up to $4 \times 10^{-4} \mathrm{~W} \mathrm{~m} \mathrm{~kg}^{-1}$ (Fig. 4d) and are on average three times larger than the values of the EKE dissipation rates at the same location. The ratio between the EKE dissipation and the EKE source, averaged over the region of eddy generation downstream from the Bimini Islands only (Fig. 4d), is 35\%, showing that about a third of the energy extracted from the mean flow by the instability processes and small-scale turbulence is locally dissipated. A smaller fraction of the EKE is converted to EPE through VBF (Fig. 4e). The ratio of the EKE converted to potential energy divided by the EKE lost to viscous dissipation gives the efficiency of the mixing ${ }^{20}$. Averaged over the domain of (Fig. 4e) in the region where $\mathrm{VBF}<0$ and the dissipation is large $\left(\langle\epsilon\rangle>0.5 \times 10^{-4} \mathrm{~W} \mathrm{~m} \mathrm{~kg}^{-1}\right)$, this efficiency is about 0.20 .

The topographic generation of centrifugal instability is remarkably sustained in time given that the current is forced through the Straits and interacts with topography consistently 

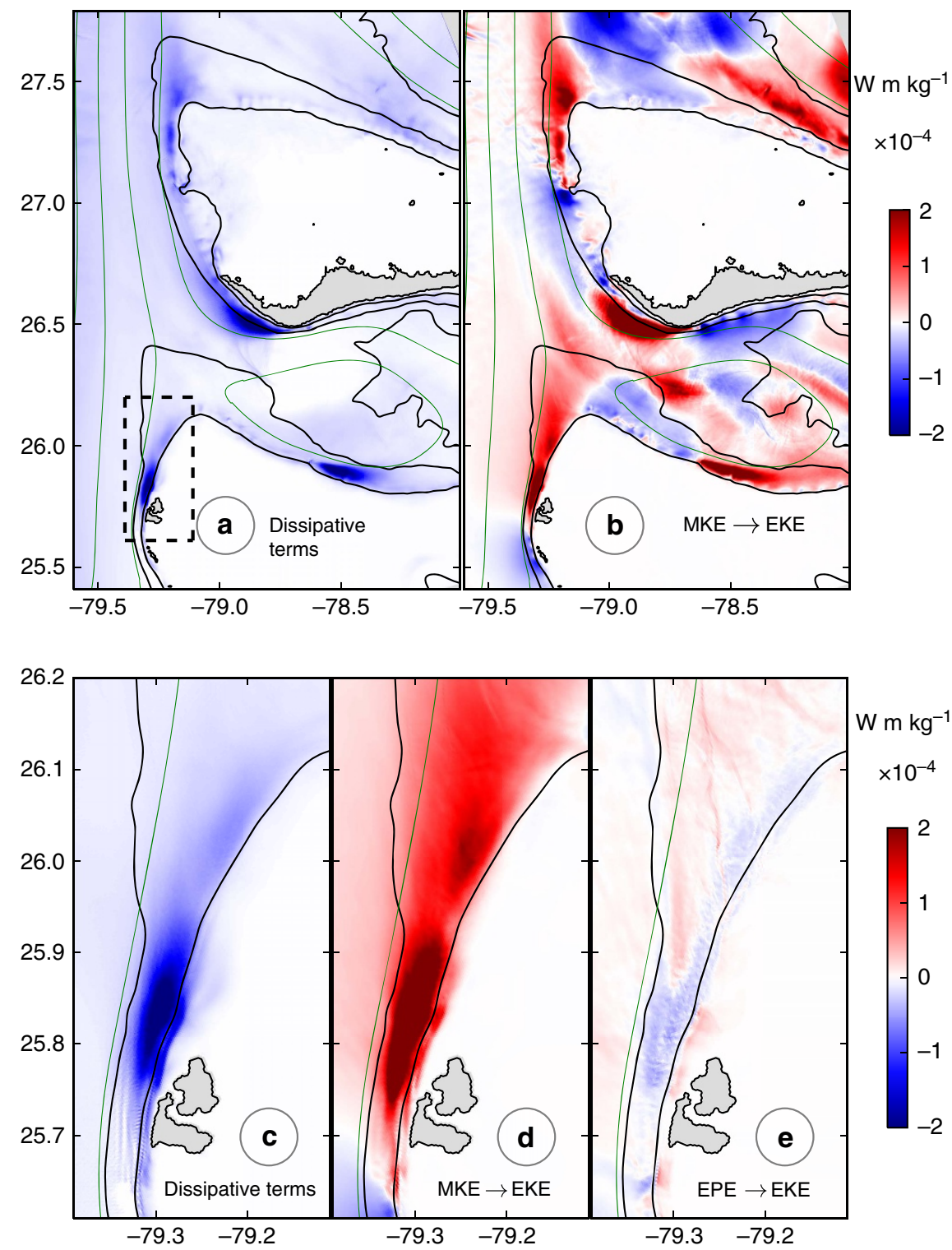

Figure 4 | Energy dissipation and mixing. Time mean depth-integrated terms of the EKE equation: (a,c) dissipative terms, (b,d) conversion from MKE to EKE, and (e) conversion from EPE to EKE. Bottom panels show a zoomed view in the region of strong generation along the Great Bahama Bank (black rectangle in a). The mean transport stream function is shown in green with a $5 \mathrm{~Sv}$ interval $\left(1 \mathrm{~Sv}=10^{6} \mathrm{~m}^{-3} \mathrm{~s}^{-1}\right)$. Units for energy rates are $\mathrm{Wm} \mathrm{kg}-1$. Topography is shown in black contours at $0,100,500$ and $1,000 \mathrm{~m}$ isobaths.

throughout the year. There is a modulation in the frequency and intensity of events following the seasonal variations of the Gulf Stream transport. The Gulf Stream transport varies by a few Sverdrups between summer where it is maximum and winter where it is minimum ${ }^{21}$. Maxima in transport are directly correlated with maxima in the intensity of the velocity shear created in the bottom boundary layer and the amplitude of the energetic dissipation induced by the centrifugal instability.

\section{Discussion}

The mechanism is generic for boundary slope currents that flow in the direction of Kelvin wave propagation (with the topography on their right in the northern hemisphere) where the PV is reduced in the bottom boundary layer ${ }^{22}$. Under these conditions, strong vertical vorticity can be generated within the bottom boundary layer that subsequently separates over complex topography and triggers intense centrifugal instability. A cyclonic eddy encountering a topographic slope will trigger the same mechanism ${ }^{23}$. This could explain, for example, the elevated levels of turbulence observed on the sides of the Bermuda Island during an interaction with a cyclonic Gulf Stream ring 24 . This could also provide another mechanism, alongside the forcing of the negative wind stress curl, responsible for the formation of the submesoscale anticyclones with large negative vorticity values $(-1.7 f)$ observed in the lee of the Hawaiian Islands ${ }^{25}$. Flows moving in the direction opposite to the Kelvin wave propagation direction, on the other hand, will generate positive vertical vorticity and eventually trigger barotropic shear instability ${ }^{14}$. But, the presence of recurrent cyclonic eddies on the cyclonic side of a current along the slope, as a results of the baroclinic or barotropic instability of the mean current, will also induce local reversals of the current and trigger the sequence of processes described here leading to centrifugal instability ${ }^{23}$. 


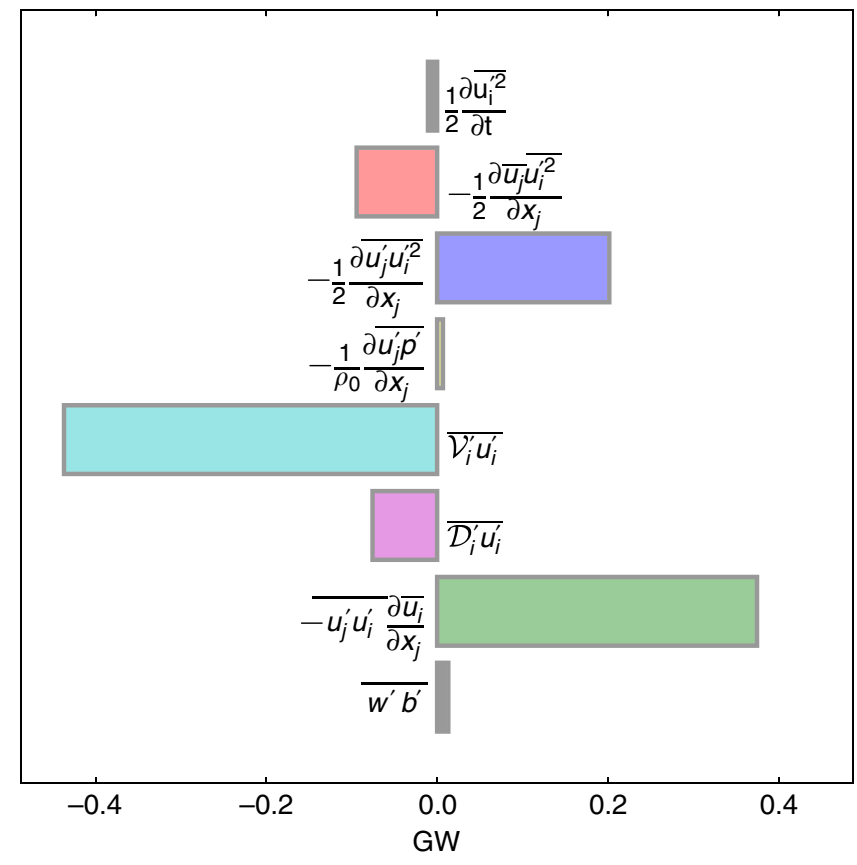

Figure 5 | Eddy kinetic energy budget. Volume integral of the EKE balance equations terms (equation 1) over the domain plotted in Fig. 4a. Units for energy rates are $\mathrm{GW}\left(10^{9} \mathrm{~W}\right)$. The main source of EKE is the conversion from mean to eddy kinetic energy due to centrifugal instability $\left(-\overline{u_{j}^{\prime} u_{i}^{\prime}} \frac{\partial \bar{u}_{i}}{\partial x_{j}}\right)$ and the main sink is the vertical mixing $\left(\overline{\mathcal{V}_{i}^{\prime} u_{i}^{\prime}}\right)$.

The sequence is much like the anticyclonic events in the California Undercurrent ${ }^{12}$, which gives birth to the submesoscale coherent vortices $\left(\mathrm{SCVs}^{26}\right)$ known as Cuddies. In the case of the Gulf Stream, the vortices produced by the instability have limited lifetimes and upscaling because they remain in the vicinity of the high shear and strain of the Gulf Stream. However, this process is also a very likely candidate for the formation of SCVs all around the globe ${ }^{27}$, including the Meddies that are formed from the Mediterranean Undercurrent along the Iberian Peninsula ${ }^{28}$, the SCVs that are formed in the Peru-Chile Undercurrent ${ }^{29}$, the SCVs observed in the northwestern Mediterranean $\mathrm{Sea}^{30}$, the SCVs that spread the Persian Gulf Outflow waters in the Gulf of $\mathrm{Oman}^{31}$, or the Labrador Sea outflow eddies that are formed along the Grand Banks ${ }^{32}$.

The volume integrated EKE dissipation and rate of conversion from mean to eddy kinetic energy are both of order $0.4 \mathrm{GW}$ $\left(10^{9} \mathrm{~W}\right)$ over the domain of Fig. 4a (Fig. 5). To evaluate the contribution of this mechanism to the global kinetic energy budget, it is interesting to scale up this value to the entire ocean and compare it to the $0.8 \mathrm{TW}\left(10^{12} \mathrm{~W}\right)$ that the wind provides to the geostrophic circulation ${ }^{33}$. The area of the ocean that has a topographic slope larger than 1 degree represents $29 \%$ of the total area ${ }^{34}$. The Gulf Stream is a highly energetic region and currents are generally weaker than the currents considered here $\left(0.5-1 \mathrm{~m} \mathrm{~s}^{-1}\right)$, such that the dissipation rates are likely to be on average an order of magnitude smaller. Assuming finally that half of the currents are flowing in the direction of Kelvin wave propagation, we arrive at a global dissipation rate of $0.05 \mathrm{TW}$, that is, in the range of $0.01-0.1 \mathrm{TW}$, which represents a significant route toward dissipation. The variability due to mesoscale eddies impinging on topography at western boundaries, islands, ridges or seamounts, is difficult to estimate and would require a more quantitative analysis using global current data from observations or global model outputs to come to a more comprehensive estimate of the energy dissipated by centrifugal instability on the boundaries.

The enhanced energy dissipation and tracer mixing due to the submesoscale turbulence, and the tracer transport by submesoscale coherent vortices, are processes that need to be quantified for their effect on the general circulation. These processes are missed by global climate models and eddy-resolving global ocean circulation models, where the grid size is generally much larger than the scale of the boundary layer, and will need to be parameterized.

\section{Methods}

Numerical simulation. The high resolution realistic simulation of the Gulf Stream is performed with the Regional Oceanic Modeling System $\left(\right.$ ROMS $\left.^{35}\right)$, which solves the free surface, hydrostatic and primitive equations. We use a nesting approach ${ }^{36}$ with successive horizontal grid nesting refinements from a parent grid resolution of $\Delta x \approx 6 \mathrm{~km}$, covering most of the Atlantic Ocean to successive nested grids with $\Delta x=2.5 \mathrm{~km}, \Delta x=750 \mathrm{~m}$, and $\Delta x=200 \mathrm{~m}$ (see domains in Fig. 1). The highest resolution solution is 4 months long and has $1,300 \times 2,300$ points in the horizontal with a resolution of $200 \mathrm{~m}$ and 100 vertical topography-following vertical levels. Vertical mixing of tracers and momentum is computed using a K-profile parameterization $^{19}$, which represents in the ocean interior small Richardson number processes and static instabilities. The effect of bottom friction is parameterized through a logarithmic law of the wall with a roughness length $Z_{0}=0.01 \mathrm{~m}$. The use of a topography-following coordinate, with significant grid stretching near the bottom such that the boundary layer mixing profile is reasonably well resolved, is of great advantage in calculating flows over complex terrain. The simulation is forced at the surface by realistic daily winds and diurnally modulated surface fluxes. Characteristics of the mean structure and variability of the simulated Gulf Stream in the Florida Straits and along the U.S. seaboard have been validated with satellite and in situ observations ${ }^{14,37}$. While one can question the details of how the model reacts to centrifugal instability, it has been shown that overall characteristics are accurate ${ }^{13}$.

Potential vorticity. The Ertel PV is defined as $q=\omega_{\mathbf{a}} \cdot \nabla b$, the dot product of the absolute vorticity $\omega_{\mathbf{a}}=f \mathbf{z}+\nabla \times \mathbf{u}$ and the gradient of buoyancy $b=-g \frac{\rho}{\rho_{0}} . f$ is the Coriolis parameter, $\mathrm{u}$ is the velocity, $\rho$ the in situ density, $\rho_{0}$ the mean reference density and $g$ the gravitational acceleration.

Eddy kinetic energy equation. The eddy kinetic energy is $\mathrm{EKE}=\frac{1}{2}\left(\overline{u^{\prime 2}}+\overline{v^{\prime 2}}\right)$, where the overline denotes a time average over the last 3 months of the simulation, and the prime denotes fluctuations relative to the time average. The EKE equation is formed by subtracting the energy equation of the mean flow (Supplementary Methods) from that of the total flow ${ }^{38}$ :

$$
\begin{aligned}
& \frac{\partial \frac{1}{2}\left(\overline{u^{\prime 2}}+\overline{v^{\prime 2}}\right)}{\partial t}+\underbrace{\frac{\partial\left(\frac{1}{2} \overline{u_{j}} \overline{u_{i}^{\prime 2}}+\frac{1}{2} \overline{u_{j}^{\prime} u_{i}^{\prime 2}}+\frac{1}{\rho_{0}} \overline{u_{j}^{\prime} p^{\prime}}\right)}{\partial x_{j}}}_{\text {Transport }}= \\
& \underbrace{-\overline{u_{j}^{\prime} u_{i}^{\prime}} \frac{\partial \overline{u_{i}}}{\partial x_{j}}}_{\text {MKE } \rightarrow \text { EKE }}+\underbrace{\overline{w^{\prime} b^{\prime}}}_{\text {EPE } \rightarrow \text { EKE }}+\underbrace{\overline{\mathcal{V}_{i}^{\prime} u_{i}^{\prime}}}_{\text {Vertical mixing }}+\underbrace{\overline{\mathcal{D}_{i}^{\prime} u_{i}^{\prime}}}_{\text {Horizontal diffusion }},
\end{aligned}
$$

where Cartesian tensor notation with summation convention has been used, $i=1$, $2, j=1,2,3 ; u_{i}$ are the horizontal components of the velocity vector $u_{j} ; u_{3}=w$ is the vertical velocity; $p$ is the pressure anomaly; $b=-\frac{g \rho}{\rho_{0}}$ is the buoyancy anomaly; $\mathcal{V}_{i}$ and $\mathcal{D}_{i}$ are the vertical mixing and horizontal diffusion terms in the horizonta momentum equations. The EKE dissipation in the model is the sum of the dissipative effects of the two latter terms. Units are $\mathrm{m}^{3} \mathrm{~s}^{-3}=\mathrm{W} \mathrm{m} \mathrm{kg}^{-1}$. The spatial structure of all terms from the mean and eddy kinetic energy balance equations are shown in Supplementary Figs 2 and 3. The volume integrated EKE budget for the domain of Fig. 4a,b is shown in Fig. 5. The volume integrated MKE budget over the same domain is shown in Supplementary Fig. 4.

Energy conversion. The conversion from mean to eddy kinetic energy $(\mathrm{MKE} \rightarrow \mathrm{EKE})$ can be due to the horizontal shear production term HRS $=-\overline{\mathbf{u}^{\prime} v^{\prime}}$. $\frac{\partial \overline{\mathbf{u}}}{\partial y}-\overline{\mathbf{u}^{\prime} u^{\prime}} \cdot \frac{\partial \overline{\mathbf{u}}}{\partial x}$ or to the vertical shear production term VRS $=-\overline{\mathbf{u}^{\prime} w^{\prime}} \cdot \frac{\partial \overline{\mathbf{u}}}{\partial z}$ (ref. 18). The conversion from eddy potential to eddy kinetic energy (EPE $\rightarrow$ EKE) corresponds to the vertical buoyancy flux $\mathrm{VBF}=\overline{w^{\prime} b^{\prime}}$, where $w$ is the vertical velocity and $b$ the buoyancy anomaly.

Code availability. The ocean model (ROMS) is available from http://www.romsagrif.org. 
Data availability. The data that support the findings of this study are available from the corresponding author upon request.

\section{References}

1. Müller, P., McWilliams, J. C. \& Molemaker, M. J. in Marine Turbulence: theories, Observations and Models (eds Baumert, H., et al.) (Cambridge Press, 2005).

2. Ferrari, R. \& Wunsch, C. Ocean circulation kinetic energy: reservoirs, sources, and sinks. Annu. Rev. Fluid Mech. 41, 253-282 (2009).

3. Capet, X., McWilliams, J. C., Molemaker, M. J. \& Shchepetkin, A. Mesoscale to submesoscale transition in the California Current System. Part II: frontal processes. J. Phys. Oceanogr. 38, 44-64 (2008).

4. Gula, J., Molemaker, M. J. \& McWilliams, J. C. Submesoscale cold filaments in the Gulf Stream. J. Phys. Oceanogr. 44, 2617-2643 (2014).

5. McWilliams, J. C. Submesoscale currents in the ocean. Proc. R. Soc. A 472, 20160117 (2016; Available at: http://rspa.royalsocietypublishing.org/content/ 472/2189/20160117)

6. Taylor, J. \& Ferrari, R. The role of secondary shear instabilities in the equilibration of symmetric instability. J. Fluid Mech. 622, 103 (2009).

7. Thomas, L. N. \& Taylor, J. R. Reduction of the usable wind-work on the general circulation by forced symmetric instability. Geophys. Res. Lett. 37, L18606 (2010)

8. D'Asaro, E., Lee, C., Rainville, L., Harcourt, R. \& Thomas, L N. Enhanced turbulence and energy dissipation at ocean fronts. Science 332, 318-322 (2011).

9. Whitt, D. B. \& Thomas, L. N. Near-inertial waves in strongly baroclinic currents. J. Phys. Oceanogr. 43, 706-725 (2013).

10. Grisouard, N. \& Thomas, L. N. Energy exchanges between density fronts and near-inertial waves reflecting off the ocean surface. J. Phys. Oceanogr. 46, 501-516 (2016)

11. Nikurashin, M., Vallis, G. \& Adcroft, A. Routes to energy dissipation for geostrophic flows in the Southern Ocean. Nat. Geosci. 6, 48-51 (2013)

12. Molemaker, M. J., McWilliams, J. C. \& Dewar, W. Submesoscale instability and generation of mesoscale anticyclones near a separation of the California Undercurrent. J. Phys. Oceanogr. 45, 613-629 (2015).

13. Dewar, W., Molemaker, M. J. \& McWilliams, J. C. Centrifugal instability and mixing in the California Undercurrent. J. Phys. Oceanogr. 45, 1224-1241 (2015).

14. Gula, J., Molemaker, M. J. \& McWilliams, J. C. Topographic vorticity generation, submesoscale instability and vortex street formation in the Gulf Stream. Geophys. Res. Lett. 42, 4054-4062 (2015).

15. Zhai, X., Johnson, H. \& Marshall, D. Significant sink of ocean-eddy energy near western boundaries. Nat. Geosci. 3, 608-612 (2010).

16. Hoskins, B. J. The role of potential vorticity in symmetric stability and instability. Q.J.R. Meteorol. Soc. 100, 480-482 (1974).

17. Cherubin, L. High-resolution simulation of the circulation in the Bahamas and Turks and Caicos Archipelagos. Prog. Oceanogr. 127, 21-46 (2015).

18. Thomas, L. N., Taylor, J., Ferrari, R. \& Joyce, T. Symmetric instability in the Gulf Stream. Deep-Sea Res. 91, 96-110 (2013).

19. Large, W., McWilliams, J. C. \& Doney, S. Oceanic vertical mixing: a review and a model with a nonlocal boundary layer parameterization. Rev. Geophys. 32, 363-403 (1994).

20. Jiao, W. \& Dewar, W. The energetics of centrifugal instability. J. Phys. Oceanogr. 45, 1554-1573 (2015).

21. Beal, L. et al. Five years of Florida current structure and transport from the Royal Caribbean Cruise Ship explorer of the seas. J. Geophys. Res. 113, C06001 (2008).

22. Benthuysen, J. \& Thomas, L. N. Friction and diapycnal mixing at a slope: boundary control of potential vorticity. J. Phys. Oceanogr. 42, 1509-1523 (2012).

23. Gula, J., Molemaker, M. J. \& McWilliams, J. C. Submesoscale dynamics of a Gulf Stream frontal eddy in the South Atlantic Bight. J. Phys. Oceanogr. 46, 305-325 (2016)

24. Hogg, N., Katz, E. \& Sanford, T. Eddies, islands, and mixing. J. Geophys. Res. 83, 2921-2938 (1978).

25. Chavanne, C., Flament, P. \& Gurgel, K.-W. Interactions between a submesoscale anticyclonic vortex and a front. J. Phys. Oceanogr. 40, 1802-1818 (2010).

26. McWilliams, J. C. Submesoscale, coherent vortices in the ocean. Rev. Geophys. 23, 165-182 (1985).
27. D'Asaro, E. Generation of submesoscale vortices: a new mechanism. J. Geophys. Res. 93, 6685-6693 (1988).

28. Bower, A., Armi, L. \& Ambar, I. Lagrangian observations of Meddy formation during a Mediterranean Undercurrent seeding experiment. J. Phys. Oceanogr. 27, 2545-2575 (1997).

29. Thomsen, S. et al. The formation of a subsurface anticyclonic eddy in the Peru-Chile Undercurrent and its impact on the near-coastal salinity, oxygen, and nutrient distributions. J. Geophys. Res. Oceans 121, 476-501 (2016).

30. Bosse, A. et al. Spreading of Levantine Intermediate Waters by submesoscale coherent vortices in the northwestern Mediterranean Sea as observed with gliders. J. Geophys. Res. Oceans 120, 1599-1622 (2015).

31. Vic, C. et al. Eddy-topography interactions and the fate of the Persian Gulf Outflow. J. Geophys. Res. Oceans 120, 6700-6717 (2015).

32. Bower, A., Hendry, R., Amrhein, D. \& Lilly, J. Direct observations of formation and propagation of subpolar eddies into the Subtropical North Atlantic. Deep-Sea Res. 85, 15-41 (2013).

33. Ferrari, R. \& Wunsch, C. The distribution of eddy kinetic and potential energies in the global ocean. Tellus 62 A, 92-108 (2010).

34. Costello, M. J., Cheung, A. \& Hauwere, N. D. Surface area and the seabed area, volume, depth, slope, and topographic variation for the world's seas, oceans, and countries. Environ. Sci. Technol. 44, 8821-8828 (2010).

35. Shchepetkin, A. \& McWilliams, J. C. The Regional Oceanic Modeling System (ROMS): a split-explicit, free-surface, topography-following- coordinate ocean model. Ocean Model. 9, 347-404 (2005).

36. Mason, E. et al. Procedures for offline grid nesting in regional ocean models. Ocean Model. 35, 1-15 (2010).

37. Gula, J., Molemaker, M. J. \& McWilliams, J. C. Gulf Stream dynamics along the Southeastern U.S. Seaboard. J. Phys. Oceanogr. 45, 690-715 (2015).

38. Harrison, D. \& Robinson, A. Energy analysis of open regions of turbulent flows: Mean eddy energetics of a numerical ocean circulation experiment. Dyn. Atmos. Ocean 2, 185-211 (1978).

\section{Acknowledgements}

We appreciate support from the Office of Naval Research (N00014-12-1-0939, N00014-12-1-0105) and the National Science Foundation (OCE-1049134) Computational resources were provided by the Pôle de Calcul Intensif pour la Mer at Ifremer, Brest, France.

\section{Author contributions}

J.G. performed the numerical simulations and led the analysis of the results and writing of the paper. J.G., M.J.M. and J.C.M. were responsible for the overall design of the experiments and interpretation of the results.

\section{Additional information}

Supplementary Information accompanies this paper at http://www.nature.com/ naturecommunications

Competing financial interests: The authors declare no competing financial interests.

Reprints and permission information is available online at http://npg.nature.com/ reprintsandpermissions/

How to cite this article: Gula, J. et al. Topographic generation of submesoscale centrifugal instability and energy dissipation. Nat. Commun. 7:12811 doi: $10.1038 /$ ncomms12811 (2016)

This work is licensed under a Creative Commons Attribution 4.0 International License. The images or other third party material in this article are included in the article's Creative Commons license, unless indicated otherwise in the credit line; if the material is not included under the Creative Commons license, users will need to obtain permission from the license holder to reproduce the material. To view a copy of this license, visit http://creativecommons.org/licenses/by/4.0/

(C) The Author(s) 2016 